Revue d'histoire de l'Amérique française

DEV REVE D.HISTOIRE DE L'AMÉRIQUE FRANÇAISE

\title{
Le tremblement de terre de 1663 : les convulsions du verbe ou la mystification du logos chez Charlevoix
}

\section{Pierre Berthiaume}

Volume 36, numéro 3, décembre 1982

URI : https://id.erudit.org/iderudit/304068ar

DOI : https://doi.org/10.7202/304068ar

Aller au sommaire du numéro

Éditeur(s)

Institut d'histoire de l'Amérique française

ISSN

0035-2357 (imprimé)

1492-1383 (numérique)

Découvrir la revue

Citer cet article

Berthiaume, P. (1982). Le tremblement de terre de 1663 : les convulsions du verbe ou la mystification du logos chez Charlevoix. Revue d'histoire de l'Amérique française, 36(3), 375-387. https://doi.org/10.7202/304068ar d'utilisation que vous pouvez consulter en ligne. 


\title{
LE TREMBLEMENT DE TERRE DE 1663: LES CONVULSIONS DU VERBE OU LA MYSTIFICATION DU LOGOS CHEZ CHARLEVOIX
}

\author{
PIERRE BERTHIAUME \\ Université d'Ottawa \\ «Quand Dieu parle, il se fait bien entendre, \\ surtout quand il parle par la voix des Tonnerres \\ ou des Terre-trembles»". \\ Hierosme Lallemand, Relation de 1663
}

«Une légère vapeur presque imperceptible s'éleva de nostre grand fleuve,» écrit Hierosme Lallemand au début de sa Relation de 1663, qui poursuit:

\begin{abstract}
et estant frappée par les premiers rayons du Soleil, deuenoit transparente, de telle sorte néantmoins qu'elle auoit assez de corps pour soustenir les deux Images que cet Astre peignoit dessus; ces trois Soleils estoient presque en ligne droite, esloignez de quelques toises les vns des autres, selon l'apparence, le vray tenant le milieu, et ayant les deux autres à ses deux costez. Tous trois estoient couronnez d'vn Arc-en-Ciel, dont les couleurs n'estoient pas bien arrestées, tantost paroissant comme celles de l'Iris, puis aprés d'vn blanc lumineux, comme si au-dessous tout proche, il y eust vne lumiere excessiuement forte. ${ }^{1}$
\end{abstract}

Ainsi s'amorce la description d'un événement resté célèbre dans les annales de la Nouvelle-France, celui du «Tremble-terre uniuersel en Canadas $)^{2}$ de 1663.

Charlevoix, qui connaissait le texte, puisera abondamment dans le récit du père Lallemand pour évoquer le séisme:

Le septiéme de Janvier de l'année suivante (écrit-il,) une vapeur presqu'imperceptible s'éleva du Fleuve, \& frappée des premiers raions du Soleil, devint transparente, de sorte néanmoins qu'elle avoit assez de corps pour soûtenir deux Parelies, qui parurent aux deux côtés de cet Astre. Ainsi l'on vit en même tems comme trois soleils, rangés sur une ligne parallele à l'Horizon, éloignés les uns des autres en apparence de quelques toises, \& chacun avec son Iris, dont les couleurs variant à

1 Hierosme Lallemand. «Relation 1663», in Relations des Jésuites, 1656-1665 (Montréal, Éditions du Jour, 1972) 5:3. (Réimpression d'Augustin Côté, 1858). À l'avenir, nous désignerons l'oeuvre par son titre, «Relation $1663 »$, suivi de la page.

2 «Relation 1663», 3.

RHAF, vol. 36, no 3, décembre 1982 
chaque instant, tantôt étoient semblables à celles de l'Arc-enCiel, \& tantôt d'un blanc lumineux, comme s'il y avoit derriere un grand feu. ${ }^{3}$

L'influence du texte de Hierosme Lallemand saute aux yeux: nous retrouvons la même terminologie, les mêmes expressions, les mêmes formules ${ }^{4}$. Il s'agit manifestement d'un cas d'intertextualité, voire de plagiat $^{5}$. Nous pourrions multiplier les exemples d'emprunts de Charlevoix au texte de la Relation de 1663 pour composer le livre VIII de son Histoire et description générale de la Nouvelle France.

Mais en même temps que nous remarquons l'influence du père Lallemand sur Charlevoix, force est aussi de constater des différences très nettes: l'historien du XVIIIe siècle ajoute des circonstances au tremblement de terre qu'on ne trouve pas dans la chronique du missionnaire jésuite, ni du reste dans la Relatio Terraemotus in Nova Francia du père Charles Simon dont Charlevoix a pu s'inspirer, et surtout, il produit un récit dont le plan n'a rien à voir avec l'organisation des deux relations de 1663: c'est que Charlevoix suit un autre texte, celui de la «Lettre CCIV» de Marie de l'Incarnation: l'anecdote de l'homme qui aperçoit soudain la terre s'ouvrir sous ses pieds ${ }^{6}$, l'expérience mystique - donc profondément intime et par là invérifiable - de Catherine de SaintAugustin ${ }^{7}$, que Charlevoix prête à Marie de l'Incarnation et plus

3 François-Xavier de Charlevoix, Histoire et description générale de la Nouvelle France avec le Journal historique d'un Voyage fait par ordre du Roi dans l'Amérique septentrionnale (Paris, Nyons Fils, 1744), I: 363. À l'avenir, nous désignerons cette oeuvre, par Histoire, suivi de la page.

4 Quelques différences: Charlevoix omet l'adjectif «légère» pour éviter un pléonasme avec l'expression "presqu'imperceptible» qui suit le terme «vapeur», il intervertit l'ordre des mots «iris» et "arc-en-ciel», il remplace la description des trois soleils par le terme scientifique «Parelies», enfin il modifie le temps des verbes en passant de l'imparfait du mémorialiste au passé simple qui convient mieux à l'objectivité supposée de l'historien.

5 Bien que la notion de plagiat ou de propriété des textes ne soit pas au XVIIIe siècle la même que la nôtre.

6 Marie de l'Incarnation, Correspondance Nouvelle édition par Dom Guy Oury (Solesme, Abbaye Saint-Pierre, 1971), 697. À l'avenir, nous désignerons cette oeuvre par le titre Correspondance, suivi de la page. Rappelons que Charlevoix avait déjà publié une Vie de la Mere Marie de l'Incarnation en 1724 (Paris, Briasson).

7 Histoire, 364. En fait l'expérience mystique en question appartient à Catherine de Saint-Augustin: dans La Vie de la mère Catherine de Saint-Augustin religieuse hospitalière de la Miséricorde de Québec en la Nouvelle France (Paris, Florentin Lambert, 1671), Paul Ragueneau décrit précisément le «pressentiment» de la religieuse hospitalière: «J'eus pour lors un présentiment assez considerable, \& comme une asseurance infaillible, que Dieu étoit prêt de punir le païs, pour les pechez qui s'y commettoient, sur-tout pour le mépris qu'on faisoit de l'Eglise. Il me sembla pour lors que Dieu étoit beaucoup irrité. Je ne pûs m'empécher de souhaiter ce châtiment quel qu'il fût; car je n'eus pour lors aucune idée de ce que ce pourroit étre. Le soir au méme instant qu'un tremblement de terre commença, je vis en esprit quatre démons, qui occupoient les quatre côtez des terres voisines, \& les secouoient fortement, comme voulans tout renverser; \& sans doute ils l'auroient fait, si une puissance superieure, qui donnoit comme le branle à tout, n'eût mis obstacle à leur volonté. Ensuite les démons me dirent qu'ils feroient leur possible pour continuer ce renver- 
globalement, l'ensemble du récit ${ }^{8}$ montre que l'historien de 1744 choisit de s'attacher plutôt à la version mystique de Marie de l'Incarnation qu'à celles, plus descriptives, de Hierosme Lallemand ou de Charles Simon.

Et cela confère au récit de Charlevoix une signification particulière: chez lui, comme chez l'Ursuline de Québec, l'événement naturel se métamorphose en témoignage de la colère divine et le séisme se mute en "héraut (...) de la divine Justice»" ${ }^{9}$ pour reprendre l'expression d'un autre historien jésuite, Joseph Acosta. En effet, chez Charlevoix, l'organisation même du texte associe directement le séisme à un crime à expier et sature l'événement d'une signification non équivoque: sous la plume de Charlevoix, le séisme devient l'expression du mécontentement de Dieu au sujet du commerce de l'eau-de-vie, doublement illicite, parce que condamné par les autorisés séculières et religieuses ${ }^{10}$.

sement, qu'il y avoit bien du monde effrayé, \& que la peur les faisoit recourir à Dieu, \& penser à leur conscience; mais qu'ils feroient bien en sorte que cela ne leur serviroit de guere.» (146-147)

8 Comme chez Marie de l'Incarnation, le récit des expériences mystiques a lieu au début du rappel du séisme chez Charlevoix, alors qu'il apparaît à la fin chez Hierosme Lallemand.

9 Joseph Acosta, Histoire naturelle et moralle des Indes, tant Orientalles qu'Occidentalles. Où il est traité des choses remarquables du Ciel, des Elemens, Metaux, Plantes \& Animaux qui sont propres de ce païs. Ensemble des moeurs, ceremonies, loix, gouvernemens \& guerres des mesmes Indiens. Composée en Castillan par Joseph Acosta, \& traduite en François par Robert Regnault Cauxois (Paris, Marc Orry, 1598), 125.

10 En cela, Charlevoix s'inspire bel et bien de Marie de l'Incarnation qui remarque dans sa lettre CCIII, datée du 12 juillet 1663, qu'au «même temps que Monseigneur notre Evêque travailloit en France pour empêcher le commerce des boissons, et apporter le remède aux désordres que quelques François causoient parmi les Sauvages, Dieu faisoit éclater icy des effets extraordinaires de sa puissance pour convertir les coupables, comme en effet il a changé des âmes toutes diaboliques, et mis en un meilleur état celles qui étoient désjà dans le bon chemin.» (Correspondance, 686) Elle reviendra à trois reprises sur l'idée dans la lettre suivante; lorsqu'elle rappelle l'expérience mystique de Mère Catherine de Saint-Augustin (Correspondance, 688), lorsqu'elle cite les propos des «Sauvages, extrêmement effraiez (qui) disoient que les arbres les avoient bien battus (et dont) quelques uns (...) disoient que c'étoient des démons dont Dieu se servoit pour les châtier, à cause des excès qu'ils avoient faits en beuvant de l'eau de vie que les mauvais François leur avoient donnée.» (Correspondance, 691) Enfin, elle reprend l'idée lorsqu'elle évoque la mésaventure de trois jeunes hommes qui allaient traiter de l'eau-de-vie et dont l'un eut une vision qui le fit rentrer en lui-même «et qui dit: il n'y a pas pourtant ici de quoi rire, nous portons des boissons aux Sauvages contre la défense de l'Eglise, et Dieu nous veut peut-être punir de notre désobéissance». (Correspondance, 694) Toutefois, l'idée que le séisme est une vengeance divine provient peut-être de Charles Simon qui écrit dans sa Relatio: «De cujus magnitudine periculi atque praesentiâ cuidam serio coram Deo cogitanti, contigit id quod ex ipsius autographo hoc loco repraesentabo cum fide translatum. Nocte, inquit quae Dominicam inter quadragesimae secundam, diemque lunae mediae volutabatur, horâ sesquidecimâ, vidi et manifeste vidi per horae dimidiae spatium, Coelum discedere, in latissimo deinde campo spiritu praesens, audivi vocem quae diceret, Respice sursum et deorsum. Cum toto corpore cohorrescerem, iterum audivi vocem Imperantis ut Respicerem. Confirmato utcumque animo paulumque resumptis viribus; vidi in lucidissima nube Angelum sagittam manu dextera librantem ad ictum: nec sane audebam prae reverentia repugnare, sed optabam ex animo tamen, et ore tacens corde tacito significabam cupere me, ut 
Voyons maintenant comment Charlevoix confère à un événement naturel cette signification divine. Nous étudierons en premier lieu la manière dont l'historien français associe le séisme à la question du commerce de l'alcool, ensuite son art du récit.

Si on observe la présentation matérielle des événements, on remarque un souci manifeste de la part de l'historien d'imbriquer étroitement la question de la traite de l'alcool à celle du tremblement de terre. Ainsi au début du récit, les titres en marge du texte, qui portent sur les doléances de Mgr de Laval au sujet de la traite

ictum sustineret, induciasque prorogaret. Respondit ille pervidens quae rogabam. Quorsum ista dilatio? Quorsum induciarum prorogatio? Respice, inquit, istos. Tum enim vero circumspexi. plurimos inter quos vidi, cognovi ex vultu duos: plenae erant manus eorum sanguine, quem proijciebant in terram, cum insigni contemptu. Intellexi sanguinem quem profundebant, alium non esse quam sanguinem Dei: cumque duos illos quos ex facie noveram turbae illi implic(at)i victimarum orco devotarum permixtos, pro viribus excusare conarer; vini Caupones, et Aquae vivae propolas, ebriosorum hominum cupiditatibus ob lucrum foede subservientes, contra severas et saepius iteratas Ecclesiae prohibitiones, et Excommunicationis fulmina, magno bonorum scandalo Rebelles; Responsum est, mensuram illorum esse cumulatam, simulatamque poenitentiam, brevique futurum ut de hypocritis illis publicum ad omnium terrorem supplicium sumeretur. Perstabam nihilominus et inducias flagitabam, optabamque ex animo poenas illorum improbitati atque obstinationi debitas in me converti. Ac mihi quidem optabiles videbantur Inferorum ignes, dummodo Dei ira cessaret. Non me tamen meus ille affectus prohibebat, quominus laetitia triumpharem, quod intelligerem Deum severam de suis inimicis, sed suae Justitiae congruentem vindictam brevi esse sumpturum.» "Relatio Terraemotus in Nova Francia, 1663", in The Jesuit Relations and allied documents Travels and explorations of the Jesuit missionaries in New France 16101791, Ed. by Reuben Gold Thwaites (New-York, Peageant Book Company, 1959, 48: 218220)

La question de la traite de l'eau-de-vie aux Amérindiens a préoccupé les autorités civiles et religieuses de la Nouvelle-France pendant bon nombre d'années. Rappelons brièvement quelques faits: un arrêt royal, daté du 7 mars 1657, condamne le commerce de l'alcool, mais l'application du règlement paraît avoir été laissée au jugement des autorités en place. Toutefois, le 31 mars 1658, Thubière de Queylus, grand vicaire sulpicien, déclare que le commerce de l'eau-de-vie avec les Amérindiens constitue un péché mortel et le 6 mai 1660, Mgr de Laval menace d'excommunication tout contrevenant à l'arrêt royal. De fait, un «engagé», Pierre Lamothe, dit Egron, sera excommunié le 18 avril 1661. À ce moment, le gouverneur Du Bois d'Avaugour paraît se rallier aux positions du clergé puisqu'un récidiviste sera puni du fouet le 10 octobre 1661 . Comme le bras séculier prend la relève du clergé, ce dernier laisse tomber en désuétude la menace d'excommunication, ce qui a peutêtre provoqué un regain d'activités chez les négociants. Résultat, Mgr de Laval rappelle la menace d'excommunication le 24 février 1662. Toutefois, pour des raisons peu éclaircies, le gouverneur n'appuie plus le clergé. De là, les doléances de Mgr de Laval à Paris.

Quelques textes à consulter sur la question: François-Vachon de Belmont, «Histoire de l'eau-de-vie en Canada», in Collection de mémoires et de relations sur l'histoire ancienne du Canada, d'après des manuscrits récemment obtenus des archives et bureaux publics en France (Québec, W. Cowan et Fils, 1840), no 8, Michel Bideaux, "Le Discours de l'ordre et le séisme de 1663", in Revue de l'Université d'Ottawa, 48 (1978): 62-83, Gustave Lanctôt, Histoire du Canada. Des origines au régime royal (Montréal, Beauchemin, 1962 (1959), I: 320-335, George F. G. Stanley, «The Indians and the Brandy trade during the ancient regime», in Revue d'histoire de l'Amérique française, 6, 4 (mars 1953): 489-505, Marcel Trudel, Histoire de la Nouvelle-France (Montréal, Fides, 1979), III: 320-330, André Vachon, «L'Eau-de-vie dans la société indienne», in La Société historique du Canada Rapport 1960: 22-32. 
de l'eau-de-vie ${ }^{11}$ intègrent singulièrement aux «plaintes» de l'évêque de Petrée le phénomène même du tremblement de terre. Mieux, la partie intitulée l' «Évêque de Petrée en porte ses plaintes au Roy" comporte deux paragraphes, dont l'un ne touche que la question du tremblement de terre. De la même façon, en déplaçant les «pressentiments» de Catherine de Saint-Augustin, ceux des Amérindiennes et en les faisant précéder l'ensemble du récit, Charlevoix donne l'illusion que le tremblement de terre n'est que la réalisation des prophéties des mystiques comme si les événements venaient confirmer les reproches ou les menaces contenus dans les extases des saintes femmes. Les événements qui suivent deviennent ainsi la preuve du courroux divin, annoncé plus haut.

Du reste, l'ensemble même du récit va dans le sens d'un message contre la vente de l'alcool aux Amérindiens. En effet, le début du texte, sur les récriminations de Mgr de Laval, et la fin du texte, sur la cessation de cet «odieux trafic, qui avoit été la premiere source de tout le mal» ${ }^{12}$ enveloppent littéralement les événements rapportés d'une introduction et d'une conclusion qui leur confèrent tout leur sens, sens que l'introduction développe avec précision. Après avoir dénoncé les «abus de la Traite de l'eau-de-vie»" ${ }^{13}$ qui apparaissent lorsque les autorités locales contreviennent aux mandements de l'évêque, Charlevoix signale un accroissement de tension entre la population et les autorités religieuses parce que celles-ci, au nom de la charité, avaient sollicité une remise de peine pour un contrevenant. "Le désordre devint extrême, note Charlevoix, on commença même à invectiver tout haut contre les Confesseurs. ${ }^{14}$ Puis, précise l'auteur, «les invectives redoublèrent» ${ }^{15}$. Enfin «le désordre alla si loin, que bientôt on n'écouta plus, ni Evêque, ni Prédicateurs, ni Confesseurs, \& que, ni les menaces de la colere Divine, ni les foudres de l'Eglise, ne purent arrêter un torrent, qui avoit rompu ses digues.» ${ }^{16} \mathrm{Et}$ c'est ce désordre que le tremblement de terre est chargé de dénoncer, de condamner, sinon de réprimer. Alors que l'évêque de Petrée est à Paris pour demander au roi des «ordres» pour «faire cesser ce commerce scandaleux» ${ }^{17}$, le ciel, au dire de l'historien, «les avoit déja prevenus, $\&$ par un de ces évenemens, qui répandent la terreur dans les ames

11 «Abus de la Traite de l'Eau-de-Vie. Conduite irreguliere du Baron d'Avaugour à ce sujet» (Histoire, 360), "Calomnies intentées à ce sujet contre l'Evêque \& les Missionnaires» (Histoire, 361), «Scandales arrivés parmi les Sauvages» (Histoire, 361) et «L'Evêque de Petrée en porte ses plaintes au Roy" (Histoire, 362).

12 Histoire, 368.

13 Histoire, 360.

14 Histoire, 361.

15 Histoire, 361.

16 Histoire, 361.

17 Histoire, 362. 
les plus libertines, on avoit déja eu la consolation dans la Nouvelle France de voir rentrer dans le devoir la plus grande partie de ceux, qui s'en étoient écartés. ${ }^{18} \mathrm{Le}$ lien entre le phénomène naturel et le message divin, dont il serait le porteur, est explicité ici. Si, chez Lallemand, le ciel et la terre ont "parlé bien des fois depuis vn an», mais un "langage aimable et inconnu», si le tremblement de terre a pu «faire trembler» les hommes en «faisant trembler la Terre» ${ }^{19}$, chez Charlevoix, le langage n'est plus inconnu. L'historien de 1744 décode le sens de l'événement et il sait reconnaître l'intervention de Dieu dans les convulsions du sol. Charlevoix délimite habilement un périmètre au séisme sur le plan de sa signification. En fait, ici, le procédé de Charlevoix paraît assez simple. Il consiste à inverser le rapport des causes et des effets, soit à faire de la cause un effet et de l'effet, une cause. Chez Hierosme Lallemand et chez Charles Simon, le séisme (la cause) provoque des sentiments de repentirs chez les pécheurs (l'effet) ${ }^{20}$; au contraire, chez Charlevoix, ce sont les péchés et plus particulièrement la désobéissance des colons au sujet de la traite de l'eau-de-vie (la cause) qui provoquent le juste courroux du ciel et la vengeance divine (l'effet). Et pour réaliser cette inversion sur le plan de la logique, Charlevoix n'a eu qu'à construire son récit de telle sorte que celui-ci débute par les expériences mystiques qui postulent la colère de Dieu et par une explication de celle-ci de façon à orienter, à hypothéquer toute l'interprétation à donner au récit qui suit ${ }^{21}$.

18 Histoire, 362.

19 Relation 1663, 2.

20 Même thèse chez Charles Simon qui remarque: «Tam inopinatus inexpectatusque casus, eoque praesertim die quo Galli nostri id unum agebant ut Bacchanaliorum tempus festis oblectationibus, comessasionibus, compotationibus, saltationibusque consumerent ne quid gravius dicam, quod castas aures offendere, meliusque reticeri, quam nominari potest; tonitru fuit ac fulmen in eorum capita coelitus vibratum, quos vel praeteritorum scelerum recordatio, vel praesentium conscientia, reos peragebant, re tamen vera magnum fuit argumentum juxta ac consilium misericordiae divinae, ut ex eventu patuit.» (Relatio Terraemotus in Nova Francia, 1663, 192). Il reprend la même idée à la fin de son récit lorsqu'il constate que «Nam sive Barbarorum conuersionem spectemus qui magno numero in Ecclesiae Matris gremium per baptismum confugerunt» (Relatio, 206) et que «Bacchanaliorum dies in dies pietatis, luctus contritionis, lacrimarumque conversi sunt privatorum preces ad multam noctem productae» (Relatio, 206).

${ }_{21}$ Ce renversement se retrouve aussi dans l'organisation même du récit qu'on peut diviser en trois parties: on trouve en premier un préambule qui introduit le phénomène, des pages 360 à 363 et qui comporte les intitulés suivants en marge: «Abus de la Traite de l'Eaude-Vie. Conduite irreguliere du Baron d'Avaugour à ce sujet», «Calomnies intentées à ce sujet contre l'Evêque \& les Missionnaires», "Scandales arrivés parmi les Sauvages», "L'Evêque de Petrée en porte ses plaintes au Roy» et "Phénomènes surprenans». Ensuite on a droit au récit proprement dit du séisme, des pages 363 à 368, qui inclut: «Prédictions d'un Tremblement de Terre», "Il commence: ses effets», parties qui permettent à Charlevoix de décrire les premières secousses et les réactions des gens, la nuit d'horreur, qui marque l'apogée du séisme, et le lendemain de cette nuit. Enfin, la troisième partie dresse le bilan des dégâts à Québec et dans les autres colonies: «Personne ne périt, \& tous se convertissent» et «Suites du Tremblement», lit-on en marge du texte, pages 368 et 369 . Or ces trois grandes divisions du récit, préambule, description du séisme, fin de celui-ci, obéissent à la 
Reste à voir comment l'art du récit permet à Charlevoix de conférer une signification providentielle au séisme et de ne pas déroger à ses devoirs d'historien. Pour ce, nous étudierons quelques aspects de la rhétorique charlevoisienne.

On peut relever chez Charlevoix bon nombre d'éléments qui appartiennent en principe à une approche scientifique, mais qui ne servent chez lui que de garanties à son interprétation des faits. Par exemple, dès le début du récit proprement dit du tremblement de terre, Charlevoix insiste pour le fonder sur «le témoignage unanime \& constant de toute une Colonie» ${ }^{22}$. Aussi il ne saurait être question de mettre en doute le fait. Mais de quel fait s'agit-il? Du tremblement de terre proprement dit ou de la signification divine de celui-ci? Or c'est ici qu'on note un subtil déplacement du sens du discours. Le «Pyrrhonisme» ${ }^{23}$ que dénonce Charlevoix porte moins sur les faits rapportés, dont l'auteur ne prétend même pas "garantir toutes les circonstances» ${ }^{24}$ que sur la dimension «extraordinaire» ${ }^{25}$ qui auréole le phénomène et qui l'accompagne. Charlevoix déplace la question; il passe du plan des faits, indiscutables, à celui du «merveilleux» ${ }^{26}$, moins vérifiable. Or si les colons de l'époque peuvent attester les faits pour les avoir vécus, en revanche ils ne sauraient devenir la caution de la signification que Charlevoix prête aux événements. Mais en entretenant une équivoque entre le niveau des faits et celui de leur signification, Charlevoix fait de la caution des premiers la garantie de son interprétation de l'histoire.

De la même façon, l'emploi d'un vocabulaire scientifique ${ }^{27}$ ou le recours à des analyses objectives au sein du récit ${ }^{28}$ ne constituent que des leurres pour donner un semblant scientifique à une vision finalement mystique du séisme. En ce sens, il n'est pas sans intérêt de constater que lors de son analyse du nombre de secousses du tremblement de terre, tout en produisant une étude objective

même structure: toujours Charlevoix fait précéder le récit proprement dit des faits de l'interprétation providentielle à leur donner. Dans le préambule, ce sont les doléances de Mgr de Laval qui permettent à Charlevoix de rendre la traite de l'alcool responsable du séisme, dans le récit, les «pressentiments» des mystiques accusent sans équivoque le commerce de l'alcool, enfin, dans la conclusion, Charlevoix commence par expliquer que «Dieu vouloit sans doute convertir les pécheurs, \& non pas les perdre» (Histoire, 368) puisque personnc n'a péri pendant les convulsions du sol.

22 Histoire, 362.

23 Histoire, 362.

24 Histoire, 362.

25 Histoire, 362.

26 Histoire, 362.

27 Par exemple les termes «Iris» et «Parelies».

28 Par exemple, lorsqu'il analyse le cas de l'île-aux-Coudres, ce qui lui permet de nier qu'elle ait été entièrement formée par une partie des Eboulements qui se serait détachée de la côte (Histoire, 369). 
du problème, Charlevoix inféode en réalité son point de vue à celui de Marie de l'Incarnation. En effet, Charlevoix constate que «quelques uns en comptèrent la nuit suivante jusqu'à trentedeux ${ }^{29}$, chiffre qu'on retrouve sous la plume de Marie de l'Incarnation ${ }^{30}$, mais qui ajoute qu'elle n'en a compté pour sa part «que six ${ }^{31}$. Ainsi le commentaire de Charlevoix, que «l'horreur de la nuit, $\&$ le trouble où l'on étoit, les firent multiplier, \& paroître plus considérables, qu'elles ne l'étoient ${ }^{32}$, répond moins au résultat d'une réflexion objective sur la situation ou à un souci de dédramatiser l'événement qu'à la volonté de tenir compte des remarques de Marie de l'Incarnation. Ce qui pouvait passer pour une analyse scientifique se révèle finalement un besoin de suivre de près le texte de la mystique de Québec.

Une preuve que Charlevoix ne poursuit pas véritablement une approche scientifique du phénomène se révèle lorsqu'il décrit la terreur des colons sans signaler qu'ils avaient cependant conscience d'être en présence d'un tremblement de terre. En effet, si dans la Relatio du père Simon les colons ont eu conscience dès les premières secousses d'être en présence d'un séisme naturel: «Tum vero ex fluctuatione terrae quae sub nostris pedibus sic tanquam instabiles cymbae iactante fluctu volutabatur; deprehendimus terraemotum subterraneis, ut conjectura erat ignibus incitatum, nec nostra nos fefellit conjectura» ${ }^{33}$, écrit le missionnaire, chez Charlevoix, au contraire, le narrateur adopte le point de vue de ceux que Charles Simon qualifie d' «ignorants» ${ }^{34}$, qui prennent panique et qui imaginent le pire. En omettant d'identifier clairement le phénomène, ce qui l'aurait réduit à ses justes proportions, Charlevoix laisse passer l'occasion de faire montre d'une attitude scientifique au profit d'un souci d'amplification de l'événement. Chez Charlevoix, le lecteur n'est pas confronté à un séisme comme chez Charles Simon, mais à des convulsions formidables du sol qui laissent une impression de démesure, d'anormalité, finalement de surnaturel. L'absence d'explication et de précision sert le dessein de Charlevoix qui cherche moins à rendre compte d'un phénomène naturel qu'à lui donner une dimension apocalyptique en laissant le lecteur au niveau des apparences et en lui faisant partager l'effroi de ceux que le séisme a terrifiés.

\footnotetext{
29 Histoire, 367.

30 Correspondance, 691-692.

31 Correspondance, 692.

32 Histoire, 367.

33 Charles Simon, op. cit., 192.

34 «At quanta in populo, causarum quae motus illos cierent Ignaro, quam improvida et tumultuosa trepidatio!» (Charles Simon, op. cit., 192).
} 
Autre aspect de la rhétorique charlevoisienne: l'insertion d'associations thématiques que la chronologie réelle des faits n'autorise pas. Dans le récit de Charlevoix, le départ de Mgr de Laval pour la France et les plaintes de celui-ci au sujet du négoce de l'eau-de-vie sont associés au tremblement de terre. Toutefois, si on analyse les faits de près, on découvre que Charlevoix modifie quelque peu la chronologie des événements pour les faire coïncider. Par exemple, Charlevoix écrit que «pendant l'automne de 1662, peu de jours aprés le depart de M. de Petrée» ${ }^{35}$, différents "phenomenes surprenants» ${ }^{36}$ eurent lieu. Pourtant le départ de Mgr de Laval pour Paris a lieu le 12 août $1662^{37}$ et les phénomènes surviennent durant l'automne, c'est-à-dire près d'un mois et demi plus tard. Ainsi le rapprochement entre le départ de l'évêque et les phénomènes liés au séisme est-il quelque peu exagéré, surtout si on tient compte du fait que le tremblement de terre n'a véritablement commencé que quatre mois et demi plus tard, soit le 7 janvier 1663. En rapprochant les deux événements dans son texte, Charlevoix crée l'illusion qu'ils sont unis dans le temps sinon logiquement; mais l'artifice parait manifeste ici ${ }^{38}$.

Autre procédé de Charlevoix pour conférer à l'événement une valeur apodictique: l'argument d'autorité. En effet, chacun des

35 Histoire, 363.

36 Histoire, 363.

37 Marcel Trudel, Histoire de la Nouvelle-France (Montréal, Fides, 1979), III: 327

38 De plus le voyage de Mgr de Laval à Paris n'a pas pour unique but de faire cesser le commerce de l'alcool. En effet, rappelle Wilfrid H. Paradis, lors de son voyage «en France en 1662, Mgr de Laval reçut l'assurance que son pouvoir ecclésiastique serait bientôt rehaussé par élévation à la dignité d'évêque titulaire» («L'Erection du diocèse de Québec et l'opposition de l'archevêque de Rouen, 1662-1674) in Revue d'histoire de l'Amérique française, IX, 4 (mars 1956): 466), ce qui implique que l'évêque de Petrée est allé solliciter auprès du roi l'érection du diocèse de Québec. Pour sa part, Claude Lessard signale aussi la question financière comme raison du voyage de Mgr de Laval: pour lui, si Mgr de Laval passe en Europe en 1662, c'est pour «amener le roi à aider de ses deniers le. Séminaire de Québec ainsi que le futur diocèse» («L'Aide financière donnée par l'Église de France à l'Église naissante du Canada", in Revue d'histoire de l'Amérique française, XV, 2 (septembre 1961): 175). En ne retenant qu'un aspect du voyage de Mgr de Laval à Paris, Charlevoix oblitère les autres dimensions du séjour de l'évêque en France et accroît par là l'importance du problème de la traite de l'eau-de-vie.

Enfin, voici la chronologie des événements, telle que reconstituée à partir des «relations» des pères Lallemand et Simon et de la Correspondance de Marie de l'Incarnation: 12 août 1662: départ de Mgr Laval.

automne 1662: apparition de phénomènes astronomiques.

7 janvier 1663: phénomène de parélie.

14 janvier 1663: second parélie.

4-5 février 1663: pressentiment d'une Algonquine nommée Catherine

expérience identique d'une autre Algonquine non identifiée (selon Marie de l'Incarnation, l'expérience de cette dernière aurait lieu le 3 février: Correspondance, 687).

5 février 1663: 17h30: premières secousses que précèdent, de peu, les «pressentiments» de Catherine de Saint-Augustin.

5-6 février: nuit d'horreur: on compte jusqu'à 32 secousses.

1er septembre 1663: éclipse de soleil.

9 septembre: fin des secousses et du séisme. 
récits de Charlevoix sur les expériences mystiques qui précèdent la description du séisme comporte le même message d'un «courroux du Ciel justement irrité contre la Nouvelle France» ${ }^{39}$. Et ce courroux, Charlevoix l'explicite par le biais de l'autorité morale de Marie de l'Incarnation, à qui il fait dire, d'ailleurs erronément ${ }^{40}$, que «la vengeance divine alloit commencer à éclatter, $\&$ que le mépris, que l'on faisoit des Ordonnances de l'Eglise, étoit sur tout ce qui allumoit la colere divine» ${ }^{41}$. Bien appuyé de l'autorité d'une femme que la ferveur populaire glorifie déjà, Charlevoix hypothèque toute la vision du tremblement de terre en indiquant, avant que ne débute la description des faits, comment observer le phénomène et quelle signification lui donner. Ainsi engage-t-il le lecteur sur la voie d'une interprétation providentielle en se fondant sur l'autorité d'une mystique en contact privilégié avec Dieu, d'une autorité donc qu'on ne saurait mettre en doute.

Finalement c'est toute l'écriture charlevoisienne qui métamorphose un phénomène naturel en affaire divine. À travers l'amplification et la divinisation des faits rapportés, Charlevoix transforme le séisme en événement surnaturel.

Qu'il y ait amplification des faits chez Charlevoix, cela saute aux yeux quand on compare le texte de l'historien de 1744 au rapport laconique du baron d'Avaugour ${ }^{42}$ ou mieux à la relation du père Lallemand. Le mouvement des toits chez Lallemand, qui «sembloient se courber en bas d'un costé, puis se renuerser de l'autre», celui des cloches qui «sonnoient d'elles-mêmes» ou celui des poutres, des soliveaux et des planchers des maisons qui «craquoient» ${ }^{43}$ 'accroissent quelque peu chez Charlevoix, qui affirme que «les toits touchoient presqu'à terre, tantôt d'un côté, \& tantôt de l'autre», que «toutes les Cloches sonnoient», que «les murs se fendoient (... et que) les planchers se détachoient, \& s'écrouloient ${ }^{44}$. Le séisme paraît plus violent en 1744 qu'en 1663. Si le père Lallemand constate «que quelques-vns ont ressenty par (les) secousses (du tremblement de terre) les mêmes souleuemens de coeur qu'ils enduroient sur l'eau» ${ }^{45}$, pour sa part, Charlevoix pré-

39 Histoire, 364.

40 Rappelons que Charlevoix confond Marie de l'Incarnation et Catherine de SaintAugustin. Voir la note numéro 7.

41 Histoire, 364-365.

42 Prosaïquement, d'Avaugour se contente de signaler: «nous avons eu un tremblement de terre qui a duré près d'un demi quart d'heure, assez fort pour nous engager à un bon acte de contrition. Il a continué de temps en temps durant neuf jours et a paru jusqu'au dernier du mois mais toujours en diminuant." (cité par Marcel Trudel, Histoire de la Nouvelle-France (Montréal, Fides, 1979), III: 330)

43 «Relation $1663 », 3$.

44 Histoire, 365 . Nous soulignons.

45 «Relation 1663», 3. 
cise que «ce qui est certain, c'est que bien des personnes ressentirent ces soulevemens de coeur \& d'estomach, \& ces tournoyemens de tête, qu'on éprouve sur Mer» ${ }^{46}$. En modifiant «quelques-vns» en «bien des personnes» et en décrivant plus précisément les manifestations physiques des maux, Charlevoix augmente le nombre des malades et la gravité de leurs malaises. Même phénomène avec la substitution du verbe «paraître» qu'on trouve chez le père Lallemand par le verbe "être»: "nostre grand fleuue de Saint-Laurens parut tout blanchastre iusques vers Tadoussac» ${ }^{47}$, écrit Hierosme Lallemand. Meilleur coloriste, Charlevoix note que les eaux «du Fleuve furent toutes blanches depuis Quebec jusqu'à Tadoussac, c'est-à-dire, l'espace de trente lieuës» ${ }^{48}$. De l'appréciation subjective d'un observateur, on passe à la description objective et précise de celui qui fait d'une évaluation, sujette à caution, une affirmation péremptoire, indiscutable, d'une précision toute mathématique. Même tendance à effacer tout doute chez Charlevoix lorsqu'il transforme «on entendoit mesme comme des voix plaintives» ${ }^{49} \mathrm{du}$ père Lallemand en «des voix plaintives augmentoient la terreur» ${ }^{50}$. Ce qui n'était qu'une présomption chez le premier, devient une certitude chez le second. Deux derniers exemples, cette fois tirés d'une comparaison entre la version de Marie de l'Incarnation et celle de Charlevoix, illustrent encore le souci de l'historien d'amplifier le phénomène. Si chez l'Ursuline de Québec, au moment des premières secousses, certains "crioient au feu, croiant que ce fût un incendie» ${ }^{51}$, chez Charlevoix, c'est «un embrasement universel» ${ }^{52}$ quel'on redoute. Enfin, alors que Marie de l'Incarnation rapporte l'expérience d'un homme «qui courut toute la nuit à mesure qu'il voyait la terre s'ouvrir» ${ }^{53}$ sous ses pieds, plus subtilement, Charlevoix écrit: «Un Homme marchant dans la Campagne apercevoit tout à coup la Terre, qui s'entr'ouvroit aupres de lui; il fuyoit, $\&$ les crevasses sembloient le suivre. ${ }^{54}$ En passant du passé simple à l'imparfait, Charlevoix généralise le fait et il donne l'illusion que l'expérience d'un individu a été celle de plusieurs, tout en dramatisant, tout en rendant plus présente l'action ${ }^{55}$.

\footnotetext{
46 Histoire, 367.

47 "Relation 1663», 4.

48 Histoire, 366.

«Relation 1663», 4.

Histoire, 366.

Correspondance, 690.

Histoire, 365.

Correspondance, 697.

Histoire, 368.
}

«Mgr J.-C. K-Laflamme, dont la science ne peut être mise en doute, écrivait en 1908, au sujet du récit de Charlevoix: "Nous croyons qu'un bon nombre des faits rapportés à ce sujet par l'historien sont exagérés. L'épouvante est une mauvaise disposition d'esprit pour faire de bonnes observations scientifiques. Ainsi, par exemple, il est difficile d'admet- 
On le voit, Charlevoix amplifie le phénomène et il lui confère finalement une dimension qui le soustrait aux lois de la nature. En passant sous silence, par exemple, la question du carnaval, pourtant présente chez Hierosme Lallemand et chez Marie de l'Incarnation, Charlevoix annule la caractère humain des événements. En fait, Charlevoix place l'homme face à son Dieu en un tête-à-tête terrible. Dans le tremblement de terre de 1663, c'est le Verbe qui se manifeste dans les convulsions titanesques de la terre et non seulement Charlevoix ne se contente-t-il pas de l'affirmer par le biais des «pressentimens», mais il suggère sans cesse la présence de l'Esprit dans les bouleversements de l'écorce terrestre. Dès les premières lignes du récit, Charlevoix dénaturalise, peut-on dire, le séisme: des «feux, sous differentes figures, toutes assez bizarres» ${ }^{56}$ volent dans l'air, "un globe de feu» ne décrit pas une orbite, mais "se promen(e) dans l'air l'espace d'environ trois lieuës» ${ }^{57}$, une "vapeur presqu'imperceptible», qui devient même transparente aux premiers rayons du soleil, possède cependant «assez de corps pour soûtenir deux Parelies» ${ }^{58}$. Dans le récit de Charlevoix, les lois physiques ne paraissent plus respectées et l'univers se trouve investi par le sacré.

Alors Charlevoix peut dépeindre un monde aux contours flous qui laisse place à l'affrontement originel entre les forces du mal et celles du bien: les eaux "ensouffrées» ${ }^{59}$, les visions, réelles

tre que des arbres se soient déracinés et replantés par la tête; que les crevasses du sol aient poursuivi les gens qui se sauvaient devant elles; que des montagnes aient glissé de la côte et soient venues se placer dans le fleuve de façon à y faire des îles; que les maisons aient oscillé autant qu'on le dit, sans qu'elles se soient renversées. Personne n'a été tué. Quant aux éboulements qu'on dit s'être produits alors, nous sommes loin de les nier; mais, d'un autre côté, nous savons de science personnelle que plusieurs de ceux qui nous ont été montrés, à diverses reprises, comme devant remonter à cette époque, n'ont aucun rapport avec ce tremblement de terre. Un bon nombre ne sont que des fragments de terrasses quaternaires encore en place.» (cité par Pierre-Georges Roy, «Le Premier grand tremblement de terre au Canada», in Le Bulletin des Recherches historiques, XXXI, 5 (mai 1925): 145-146).

56 Histoire, 363.

57 Histoire, 363. Selon Alphonse Gagnon, les «globes de flammes» sont en fait des météores et il précise: «il n'est pas rare, d'ailleurs, que des tremblements de terre soient précédés de phénomènes de ce genre», du reste connus depuis l'époque même «de Pausanias» (Études archéologiques et variétés, Montréal, Beauchemin, 1913, 116).

58 Histoire, 363.

59 Histoire, 366 et 368 . Pourtant Charlevoix pouvait trouver une explication scientifique au phénomène dans la Relatio Terraemotus in Nova Francia de Charles Simon: «His quae consulto paulo fusius exposui mature consideratis, quanta fuerit terrae subversio ex eo facillime colligi potest, quod talis tantusque fluvius emotis ab imo fundo, et in eum effusis terrae visceribus ac sulphurariis fodinis, aquis sulphuris infusi copia dilutis, non ad breve temporis spatium, sed ad octiduum totum mutavit colorem, sulphureum induit, tenuitque constanter. Magnum pretiosorum quae terra celat, seu periculorum, seu metallorum, aurique praesertim quod tantopere sitiunt homines ab exordio mundi ad hanc diem irreperti argumentum. Sed haec nos obiter, alij viderint, redeamus in viam.» (The Jesuit Relations, New-York, Peageant Book Company, 1959, 48: 204) 
ou imaginaires, de «Spectres» et de «Fantômes» ${ }^{60}$, les phénomènes auditifs étranges constituent autant d'éléments sur lesquels insiste Charlevoix et qui jettent le lecteur au coeur d'un univers aux confins mal définis, au coeur d'un univers qui touche à la fois aux domaines infernal et éthéré. Sous la plume de Charlevoix, le séisme devient l'occasion d'opposer aux puissances ténébreuses le Dieu de lumière, qu'évoque du reste l'auteur lorsqu'il rappelle que, dans la Bible, le Seigneur en usa ainsi «à l'égard des Ninives, qui parerent le coup, dont ils étoient menacés, par une penitence exemplaire ${ }^{61}$. Manifestement, les convulsions de la terre ne constituent que la manifestation d'un affrontement infiniment plus grand qui oppose le bien au mal, le Ciel aux puissances infernales. Pour l'historien jésuite, "cet évenement, à en juger par l'effet, qu'il produisit, a tout l'air d'un avertissement du Ciel, \& (...) la conduite ordinaire de la Providence en pareilles occasions, est de faire avertir les Coupables que la Justice divine est prête à lancer la foudre ${ }^{62}$. L'évocation des textes sacrés, la présence de signes appartenant au Malin, enfin l'intervention du Ciel importent dans le récit un logos qui questionne le sens même du salut de l'humanité. Ce qui est en jeu dans le séisme de 1663, au-delà de la question controversée du commerce illicite de l'alcool, c'est la lutte éternelle, dans un monde convulsé, de Dieu et de l'ange déchu, et le souffle qui passe au-dessus de la malheureuse colonie appartient à l'infini. Ici, le récit de Charlevoix quitte le champ de l'histoire pour celui de la mythologie.

En cela se trouvent sans doute les limites du texte de l'historien,mais aussi sa grandeur ${ }^{63}$.

60 Histoire, 366. Encore ici, Charles Simon paraît nettement plus méfiant que Charlevoix au sujet des visions des gens. Par exemple, alors qu'il rapporte que les Amérindiens virent au ciel un jeune homme avec une torche, il ajoute prudemment «sive illa vere viderint, sive ut fit in rebus turbulentis, se vidisse putaverint» (op. cit., 48: 206).

61 Histoire, 363.

62 Histoire, 363.

63 Est-il besoin de rappeler la fortune de l'interprétation charlevoisienne? Si les historiens paraissent plutôt sceptiques à l'égard de l'explication que Charlevoix propose du séisme de 1663, en revanche, les rédacteurs des manuels scolaires abondent dans le sens de l'historien jésuite. Par exemple, Gustave Lanctôt établira un lien de cause à effet entre la recrudescence du commerce de l'alcool et le séisme (Histoire du Canada; Des origines au régime royal, Montréal, Beauchemin, 1960, 329) et C. H. Laverdière intégrera des extraits du récit de Charlevoix dans son Histoire du Canada à l'usage des maisons d'éducation (Québec, Presses d'Augustin Côté, 1869, 83). 